This is the submitted version of the following article:

Marcelo G.A., Montpeyo D., Novio F., Ruiz-Molina D., Lorenzo J., Oliveira E.. Luminescent silicon-based nanocarrier for drug delivery in colorectal cancer cells. Dyes and Pigments, (2020). 181. 108393: - . 10.1016/j.dyepig.2020.108393,

which has been published in final form at https://dx.doi.org/10.1016/j.dyepig.2020.108393 @ https://dx.doi.org/10.1016/j.dyepig.2020.108393. This manuscript version is made available under the CC-BY-NC-ND 4.0 license http://creativecommons.org/licenses/by-nc-nd/4.0/ 


\title{
Luminescent silicon-based nanocarrier for drug delivery in colorectal cancer cells
}

\author{
Gonçalo A. Marcelo a, David Montpeyo b, Fernando Novio c,d, Daniel Ruiz-Molina c, Julia Lorenzo b,**, \\ Elisabete Oliveira a,e, ${ }^{\circ}$ a \\ a BIOSCOPE Group. LAQV@REQUIMTE. Chemistry Department. Faculty of Science and Technology, University NOVA of Lisbon, Caparica Campus, 2829-516, Caparica, Portugal \\ b Institut de Biotecnologia i Biomedicina, Departament de Bioquímica i de Biologia Molecular, Universitat Autònoma de Barcelona, Bellaterra, Barcelona, Spain \\ c Catalan Institute of Nanoscience and Nanotechnology (ICN2), CSIC and The Barcelona Institute of Science and Technology, Campus UAB, Bellaterra, 08193, Barcelona, Spain \\ d Departament de Quimica, Universitat Autònoma de Barcelona, Campus UAB, Cerdanyola del Vallès, 08193, Barcelona, Spain \\ e PROTEOMASS Scientific Society, Rua dos Inventores. Madam Parque, Caparica Campus, 2829-516, Caparica, Portugal
}

A R T I C L E I N F O Keywords: Luminescence, Silicon quantum dots, Mesoporous silica nanoparticles, Drug delivery, Colorectal cancer cells, Doxorubicin

A B S T R A C T Nanocarriers sensitive to exogenous or endogenous stimuli emerged as an attractive alternative to target drug delivery, with inorganic silica mesoporous nanoparticles (MNs) playing a core role in the development of a new generation of non-toxic and tuneable nanocarriers. A sensitive nanovector (NANO1) comprising luminescent silicon quantum dots (SiQDs) and functionalized with MNs was synthesised and loaded with doxorubicin (DOX). NANO1 nanoparticles have a size of $74 \pm 10 \mathrm{~nm}$ and DOX loading percentages of ca. 43\%. As a control sample, a similar nanocarrier (NANO2), without SiQDs, was also synthesised and loaded with DOX. Release profile studies, in PBS, revealed the strong NANO1@DOX pH-dependant behaviour, with a pH 5.0 favouring the release of DOX to percentages of ca. 70\%. Cytotoxicity assessments of both free and DOX-loaded nanocarriers were evaluated in human cell lines of colon, revealing both free drug and drug-loaded nanoparticles to be concentration-dependent.

\section{Introduction}

Colorectal cancer (CRC) is the second most common cancer and the fourth cause of cancer deaths in the world, with nearly 1.4 million new cases diagnosed in 2012 [1]. The development of new nanotech-based carriers to increase (i) specificity to target tumour cells, (ii) delivery capacity to the tumour site, therefore maximising the efficacy of the therapy and (iii) minimising undesirable side effects, is crucial [2,3]. Nanocarriers sensitive to exogenous or endogenous stimuli emerged as an attractive alternative to target drug delivery, but only a few stimuli-responsive nanosystems have reached clinical stage due to their complex design and challenging synthetic scale-up [4]. From all nanoparticles systems that have been used as drug delivery agents, luminescent inorganic silica mesoporous nanoparticles (SiQDsMNs) emerged as a promising multifunctional new generation of nanocarriers. Their use as drug delivery devices is due to their low toxicity, high specific surface area, large pore volume, tunable pore structures, and size [5-7]. Usually, to facilitate their tracking in biological systems, nanocarriers are labelled with a luminescent dye or, in this case, a luminescent inorganic quantum dot. Based on previous studies, silicon quantum dots are non-toxic biocompatible emissive materials, with numerous in vivo experiments revealing that silicon degrades to silicic acid, that is later excreted in urine [8-11]. The first reports on silicon nanocrystals were published two decades ago $[12,13]$. However, there are persistant challenges on the working procedures for this type of material [14], as i) the feasible preparation of high-quality colloidal silicon nanocrystal, ii) the easy control of their size and optical properties and iii) the avoidance of surface oxidation on silicon quantum dots. Regarding the available synthetic methods for SiQDs, most methods reported to date rely on relative harsh conditions (strong acids, toxic reagents, organic solvents, high temperature, inert atmosphere), specialised equipment, complex experimental procedures, not easily approachable by non-experts and with scaling up problems [14]. For a successful application in biological systems, SiQDs must be water-soluble, avoiding then any post-treatment and expensive processes. Recently, Y. Zhong and co-workers reported an aqueous synthetic method to prepare fluorescent SiQDs under microwave irradiation at 160 $\$$ [15]. Later, the same group developed a photochemical strategy towards the synthesis of large-quantity of SiQDs under UV irradiation [16]. Fabrication of ultrabright water-dispersible SiQDs through a designed chemical surface modification [17], as well as, one-step synthesis of waterdispersible SiQDs for time-resolved imaging of living cells [18] and for selective sensing of heparin and cell imaging [19] were already reported by other authors. Based on these previous studies, in this work, SiQDs were performed in a one-step synthesis in water using (3aminopropyl) trimethoxysilane (APTMS) and sodium ascorbate (SA) [18]. Despite all reported synthetic methods of silicon quantum dots in water [15-19], nanocarriers resulting from the combination of silicon quantum dots and silica mesoporous nanoparticles are yet scarce $[20,21]$. Additionally, from all nanocarriers developed to date that are under the preclinical stage for CRC [22-35], none combines luminescent quantum dots (silicon quantum dots) and mesoporous silica nanoparticles. Taking this into account, herein it is presented, for the first time, the synthesis of a sensitive nanocarrier combining silicon quantum dots and silica mesoporous nanoparticles and consequent surface functionalization, as well as its cytotoxic evaluation in colorectal cancer cells and drug delivery capacity (via pH stimuli) in saline buffer (at $\mathrm{pH} 7.4$ and 5.0). To surmise, the delivery of anti-cancer drugs in fully non-toxic materials (non-use of toxic luminescent QDs) comprising SiQDs and mesoporous matrixes, has, according to literature [36,37], never been reported. Thus, the novelty of the work stands, despite a recent report by the authors on the sole toxicity of mesoporous SiQD-based systems [21], on the foremost development of similar nanosystems in the controlled delivery of DOX in specific cancer colorectal cell lines, for future applications as building blocks in targeted nanotherapies. 


\section{Experimental}

\subsection{Chemicals and starting materials}

(3-aminopropyl)triethoxysilane (APTES, $\mathrm{H}_{2} \mathrm{~N}\left(\mathrm{CH}_{2}\right)_{3} \mathrm{Si}_{(}\left(\mathrm{OC}_{2} \mathrm{H}_{5}\right) 3,99 \%$ ), (b)-sodium, L-ascorbate ( $\mathrm{SA}, \mathrm{C}_{6} \mathrm{H}_{7} \mathrm{NaOO}_{6},>98 \%$ ), tetraethyl orthosilicate (TEOS, $\left.\mathrm{SiC}_{8} \mathrm{H}_{20} \mathrm{O}_{4}, 98 \%\right)$, ammonia $\left(\mathrm{NH}_{3},>99.9 \%\right)$, cetyltrimethylammonium bromide (CTAB, $\left.\mathrm{C}_{19} \mathrm{H}_{42} \mathrm{BrN}, \quad>98 \%\right)$, (3mercaptopropyl)trimethoxysilane (MPTS, 95\%), aldrithiol-2 (98\%) (2,20-Dipyridyl disulfide), cysteamine (>98\%), were purchased from Sigma-Aldrich. Ammonium nitrate ( $\left.\mathrm{NH}_{4} \mathrm{NO}_{3}, 99.9 \%\right)$ was purchased from Alfa Aesar. Methanol ( $\mathrm{MeOH}$ ) and ethanol (EtOH) were produced by Carlo Erba Reagents. All solvents and reagents were of analytical reagent grade and were used as received.

\subsection{Synthetic procedures}

Silicon quantum dots (SiQDs) were prepared by adding $2 \mathrm{~mL}$ of 3-aminopropyltriethoxysilane (APTES) to $4 \mathrm{~mL}$ of miliQ water, followed by the addition of $4 \mathrm{~mL}$ of an aqueous solution of sodium ascorbate $(0.1 \mathrm{M})$. The final mixture was stirred for $2 \mathrm{~h}$ at $50{ }^{\circ} \mathrm{C}$, leading to the formation of green emissive SiQDs, as reported in literature [18]. To the previous mixture (SiQDs) was added $10 \mathrm{~mL}$ of an aqueous solution of CTAB $(0.1 \mathrm{M})$ and kept under stirring for $30 \mathrm{~min}$ at $50{ }^{\circ} \mathrm{C}$. Ammonia $(500 \mu \mathrm{L})$ was added and the mixture was stirred for a further $10 \mathrm{~min}$. Then, $240 \mu \mathrm{L}$ of TEOS was added dropwise and the final mixture left under stirring for $2 \mathrm{~h}$ at $70{ }^{\circ} \mathrm{C}$. After $2 \mathrm{~h}, 3-$ Mercaptopropyltrimethoxysilane $(1 \mathrm{~mL})$ was added and after $30 \mathrm{~min}$ of stirring the resulting nanoparticles were washed with water and centrifuged, resulting in the nanoparticles SiQDsMNsSH. The SiQDsMNsSH nanomaterial $(200 \mathrm{mg})$ was treated with a methanol solution containing the precursor PDEA (2-(pyridyldisulfanyl) ethylamine) $(200 \mathrm{mg}$ ), which was previously synthesised accordingly to Refs. [38,39], for $24 \mathrm{~h}$ under stirring. The resulting nanomaterial SiQDsMNsS-S-NH2 (NANO1) containing the 2-(propyldisulfanyl) ethylamine linker was centrifuged, washed several times with methanol and dried. Template removal was achieved by solvent extraction, by resuspending the nanoparticles in a $20 \mathrm{~mL}$ methanolic solution containing $60 \mathrm{mg}$ ammonium nitrate, that was stirred for $2 \mathrm{~h}$ at $60{ }^{\circ} \mathrm{C}$. The procedure was repeated twice and the nanoparticles centrifuged and washed in water. As a control sample the same synthesis was repeated, but without the addition of silicon quantum dots (SiQDs), resulting in MNsS-S-NH2 (NANO2) nanoparticles.

\subsection{Nanoparticles characterisation}

UV-Vis absorption spectra were recorded on a Jasco V-650 spectrophotometer and a NANODROP ND-1000; and Fluorescence emission in a HORIBA Scientific FLUOROMAX-4 spectrofluorimeter from Proteomass- Bioscope facility (Portugal). Infrared spectra were acquired in a FTIR Spectrometer Spectrum two, UATR TWO PERKIN ELMER. Hydrodynamic size and zeta potential were obtained by dynamic light scattering, by dispersion of the nanoparticles in miliQ water, in a Malvern Nano ZS Zetasizer instrument with a $633 \mathrm{~nm}$ laser diode, from Proteomass - Bioscope facility (Portugal). Transmission electron microscopy (TEM) images were obtained in a JEOL JEM 2010F operating at $200 \mathrm{kV}$. TEM images were collected using a multiscan camera and Digital Micrograph software from Gatan (CCD Gatan 895 USC4000). Surface area and pore size distribution were obtained from $\mathrm{N} 2$ adsorption/desorption isotherms at $77 \mathrm{~K}$, in a Micromeritics ASAP 2010 (Accelerated Surface Area and Porosimetry), at the Laboratory of Analysis from FCT - UNL. Specific surface areas (SBET) were estimated with the BET method [40]. XRD spectra were acquired with RIGAKU MiniFlex II X-ray diffractometer equipped with a Cu-Ka source (30 $\mathrm{KV} / 15 \mathrm{~mA}$ ) for $2 \theta$ angles ranging from $3 \circ$ to $80^{\circ}$. The fluorescence quantum yield of SiQDs was measured using as standard fluorescein $(\varphi$ $O$ 0.79) [41] in ethanol. The value of fluorescence quantum yield was corrected accordingly with the different refraction indexes.

\subsection{Doxorubicin loading and in vitro release studies}

To a solution of doxorubicin (DOX) $(1 \mathrm{mg} / \mathrm{mL}$ ), previously prepared in phosphate buffer (PBS) pH 7.4, were added 24 mg of NANO1 (SiQDsMNsS-S-NH2) or NANO2 (MNsS-S-NH2) nanoparticles and kept under stirring for $24 \mathrm{~h}$ at room temperature. After that, the sample was centrifuged and washed four times with PBS 7.4. The supernatants were quantified by absorption in the NANODROP ND-1000, from which were obtained the encapsulation efficiency $(\mathrm{EE} \%)$ and the loading capacity $(\mathrm{mg} / \mathrm{g})$, determined by the following equations (toox: the total amount of DOX/nanoparticles fDox: the amount of free DOX/nanoparticles) [42]:

$$
\begin{aligned}
& E E(\%)=\frac{t_{D O X-f_{D O X}}}{t_{D O X}} \times 100 \% \\
& \text { loading capacity }(\mathrm{mg} / \mathrm{g})=\frac{t_{D O X}(\operatorname{mg})-f_{D D X}(m g)}{\text { amount of nanoparticles }(\mathrm{g})}
\end{aligned}
$$

The in vitro doxorubicin release from the encapsulated NANO1@DOX and NANO2@DOX was performed by suspending the nanoparticles $(10 \mathrm{mg}$ ) in a $2 \mathrm{~mL}$ solution of PBS 7.4 and PBS 5.0. All suspensions were stirred and kept at $37 \circ \mathrm{C}$. Aliquots were collected at different times and the released doxorubicin quantified by absorption in the NANODROP ND- 1000.

\subsection{Cell culture}

Colon cancer cell lines HT29 and HCT116 were obtained from the ATCC (American Type Culture Collection). All cell cultures were routinely maintained in Dulbecco's modified Eagle's medium (DMEM) supplemented with $10 \%$ foetal bovine serum (FBS) in standard growth conditions $\left(37^{\circ} \mathrm{C}, 10 \% \mathrm{CO} 2\right)$ 


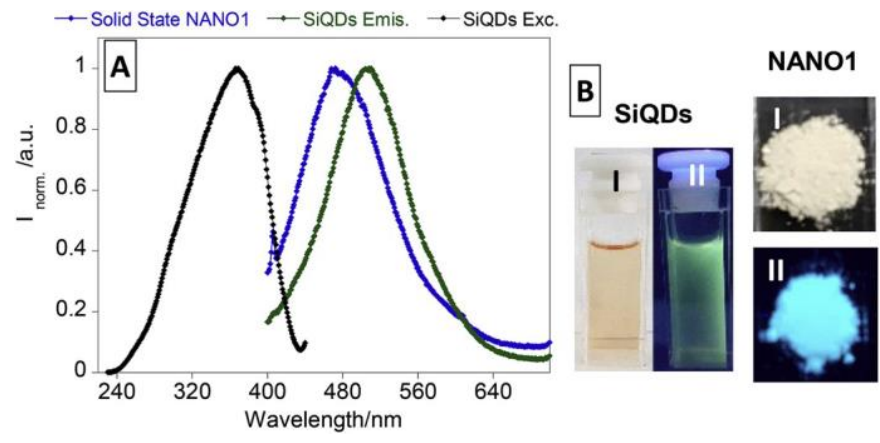

Fig. 1. (A) Emission and excitation spectra on SiQDs and solid-state emission spectrum of NANO1 nanoparticles ( $\lambda$ exc $1 / 4370 \mathrm{~nm}, \lambda$ em $1 / 4510 \mathrm{~nm}$ ). (B) Naked-eye (I) and under UV lamp (II) ( $\lambda_{\text {exc } 1 / 4} 365 \mathrm{~nm}$ ) images of Silicon quantum dots in solution and of the NANO1 nanoparticles in solid state. (For interpretation of the references to color in this figure legend, the reader is referred to the Web version of this article.)

\subsection{Cytotoxicity and intracellular accumulation of the nanoparticles}

The cytotoxicity of free nanoparticles NANO1 and NANO2, as well as those loaded with doxorubicin (NANO1@DOX; NANO2@DOX) was tested in human cancer colon cells using the PrestoBlue assay. Cells were seeded into a 96-well plate at a cell density of $3.0 \times 10^{3}$ cell/well and incubated for $24 \mathrm{~h}$ before nanoparticles were added at a concentration from 1 to $300 \mu \mathrm{g} / \mathrm{mL}$. After $72 \mathrm{~h}, 20 \mu \mathrm{L}$ of prestoBlue reagent were added to each well and the plates were further incubated for $4 \mathrm{~h}$ at $37{ }^{\circ} \mathrm{C}$. The effect on cell viability was measured using a multilabel plate reader (Victor 3 PerkinElmer). Its fluorescence was quantified after excitation at $\lambda{ }^{\prime} \mathrm{O} 531 \mathrm{~nm}$ and collection at $\lambda{ }^{\prime} \mathrm{O} 615 \mathrm{~nm}$, while its absorbance was monitored at $\lambda=570 \mathrm{~nm}$. Cell cytotoxicity was evaluated in terms of cell growth inhibition in treated cultures and expressed as a percentage of the control conditions. Each experiment was repeated at least three times, and each concentration was tested in at least three replicates. For cellular accumulation determination, $10^{5} \mathrm{HT} 29$ cells were plated in $35 \mathrm{~mm}$-cell culture dishes and were allowed to grow overnight. Cells in exponential growth were incubated, for $24 \mathrm{~h}$, with $50 \mu \mathrm{g} / \mathrm{mL}$ of each nanoparticle (untreated cells and cells treated with $1.75 \mu \mathrm{M}$ of doxorubicin were used as a control). After incubation, cells were washed twice with PBS and lysed with PBS containing $1 \%$ SDS. Fluorescence intensity of the lysates was measured in a Jasco FP-8200 spectrofluorometer at $\lambda_{\mathrm{ex}}=370 \mathrm{~nm} / \lambda_{\mathrm{em}}=$ $499 \mathrm{~nm}$ and $\lambda_{\mathrm{ex}}=470 \mathrm{~nm} / \lambda_{\mathrm{em}}=589 \mathrm{~nm}$. Initial fluorescence intensity was measured from $50 \mu \mathrm{g} / \mathrm{mL}$ of each nanoparticle in PBS containing $1 \%$ SDS (Sodium Dodecyl Sulphate), and the uptake ratio was calculated for every fluorescence-emitting nanoparticle. 2.7. CLSM in vitro imaging of nanoparticles-treated cells Cellular uptake experiments were performed on HT29 cell line. Cells were seeded at a density of about $2 \times 10^{4}$ cells per plate on $14 \mathrm{~mm}$ glass-bottom microwell dishes (MatTek, corp). After $24 \mathrm{~h}$, the cells were treated with $25 \mu \mathrm{gg} / \mathrm{mL}$ of NANO1@DOX and NANO2@DOX, and incubated for additional $24 \mathrm{~h}$. Cells were washed three times with cell media, and cell nuclei and membrane were stained with Hoechst or CellMask respectively for $15 \mathrm{~min}$. Cell images were taken using a laser scanning confocal microscope (LSCM) SP5 (Leica).

\section{Results and discussion}

\subsection{Synthesis and characterisation}

Luminescent Silicon quantum dots (SiQDs) were synthesised in water and in open-air conditions at $50{ }^{\circ} \mathrm{C}$ for $2 \mathrm{~h}$. APTES and sodium ascorbate were used as silica source and as stabiliser/reducer. Green luminescent SiQDs showed excitation and emission maximum bands centred at ca. $370 \mathrm{~nm}$ and ca. $510 \mathrm{~nm}$, respectively (Fig. 1). Additionally, the SiQDs presented a hydrodynamic size of $3.6 \mathrm{~nm}$, a zeta potential of $-3.6 \mathrm{mV}$ and a fluorescence quantum yield of 13\%. The resulting SiQDs were covered with a layer of mesoporous silica nanoparticles (SiQDsMNs), functionalized with a layer of MPTS (SiQDsMNsSH) and the 2-(propyldisulfanyl) ethylamine linker finally added to the nanoparticles, leading to the final nanoparticles NANO1 (SiQDsMNsS-S-NH2) as depicted in Scheme 1 and Fig. 1. As control sample for the in vitro assays in colon cancer cell lines, the same nanocarrier without silicon quantum dots was also synthetized (NANO2). The final nanocarrier NANO1 was characterized by solid-state emission, TEM, FTIR, zeta potential, N2 isotherms and XRD. Moreover, the different coatings were confirmed by zeta potential, FTIR and N2 isotherms. As can be seen in Fig. 1B, NANO1 was obtained as a white blue emissive powder with a maximum emission band centred at $475 \mathrm{~nm}$. As reported by Dasog and co-workers [37,43,44], the emission shift from green to blue upon functionalization of the SiQDs with mesoporous silica nanoparticles is attributed to the presence of nitrogen and oxygen sources leading the formation of silicon oxynitride type species. Based on TEM images NANO1 present a spherical shape with a size of $74 \pm 10 \mathrm{~nm}$ (see Fig. 2A and B). The presence of silica element was also confirmed in the EDX spectra, depicted in Fig. 2C. To investigate the porosity of the materials, nitrogen adsorption/ desorption isotherms were performed (Fig. 2E). The luminescent unfunctionalized, SiQDsMNs, showed a BET surface area of $677.7 \mathrm{~m} 2 / \mathrm{g}$ and pore size of $4.1 \mathrm{~nm}$ (BJH model). The isotherm can be classified as a type IV isotherm with an adsorption step behaviour at P/PO around $0.3-0.35$, characteristic of mesoporous materials [45]. With the addition of further coatings, it is notable a decrease of surface area from SiQDsMNsSH to SiQDsMNs-S-S-NH2 (NANO1), with $96.9 \mathrm{~m} 2 / \mathrm{g}$ falling to $13.5 \mathrm{~m} 2 / \mathrm{g}$, respectively. Due to the decrease of surface area, and so of the mesoporosity of the system, the consequent release through the pores of the silicon quantum dots is prevented, and the luminescent characteristics of the nanocarrier maintained. The XRD spectra (Fig. 2F) showed a broad peak at ca. 22० (SiQDsMNsSH, NANO1, NANO2), which corresponds to the amorphous peak of silica oxide. Zeta potential showed that the NANO1 had a surface charge of $44.8 \pm 0.8 \mathrm{mV}$, while that of SiQDsMNs and SiQDsMNsSH was of $28.6 \pm 0.6$ 
$\mathrm{mV}$ and $40.6 \pm 0.7 \mathrm{mV}$, respectively (Fig. 3). The above described values were obtained in miliQ water and at neutral $\mathrm{pH}$ of 7.0. From the general point of view, the increase of coatings functionalizing the surface stabilises the nanocarrier, turning it more positive. The different coatings were also verified by infrared spectroscopy. The FTIR spectra (Fig. 3) of SiQDsMNsSH and NANO1 show the characteristic bands of the silica structure, with a peak at $1028 \mathrm{~cm}$ l corresponding to the Si-O-Si vibrations (asymmetric stretching). The absence of a peak at ca. $965 \mathrm{~cm}^{-1}$ for the SiQDsMNsSH nanoparticles shows that $-\mathrm{OH}$ groups on the surface of the nanosilica have completely reacted with MPTS. Covering by MPTS can also be confirmed by the peaks at $1117 \mathrm{~cm}$ ( 1 (-Si-O-C-stretching), $1237 \mathrm{~cm}^{-1}\left(\mathrm{Si}-\mathrm{CH}_{2}-\mathrm{R} \mathrm{stretch}\right)$ and $2932 \mathrm{~cm}^{-}$ ${ }^{1}$ (CH stretch). Moreover, the amino linker was confirmed by the peak at $1521 \mathrm{~cm}$ l, $1627 \mathrm{~cm}$ l corresponded to the groups ( $\mathrm{C}-\mathrm{N}$ stretch) and ( $\mathrm{R}-\mathrm{NH}_{2}$ in-plane bend), respectively.

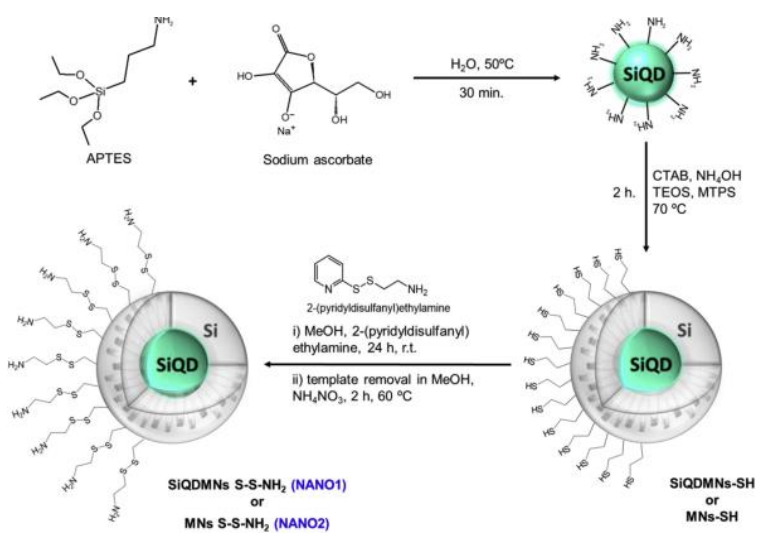

Scheme 1. General synthetic pathway of the NANO1 and NANO2 nanoparticles.
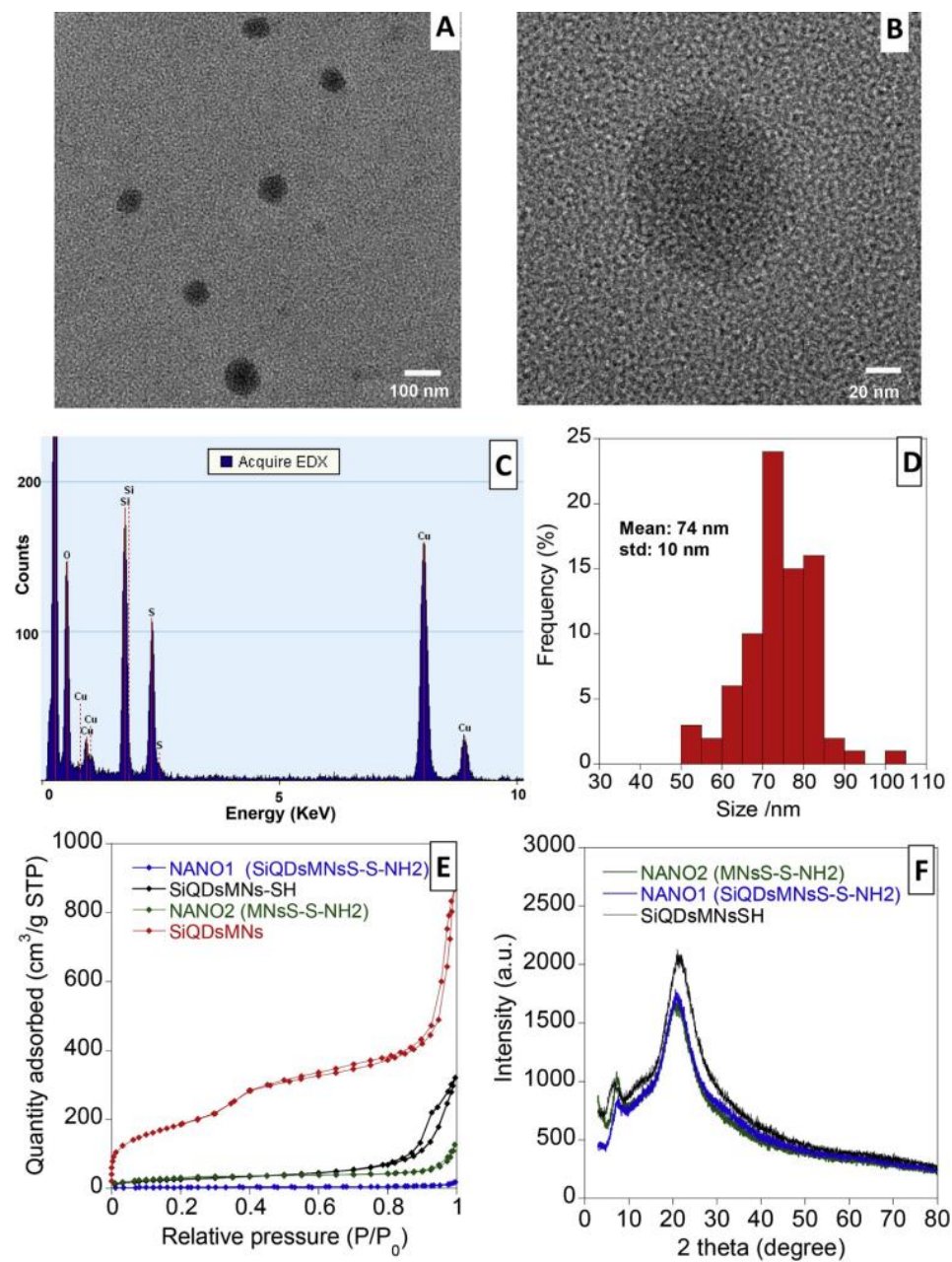

Fig. 2. TEM images (A, B), EDX spectrum (C) and size distribution (D) of NANO1 nanoparticles. (E) Nitrogen adsorption -desorption isotherms of NANO1, SiQDsMNsSH, NANO2 and SiQDsMNs nanoparticles. (F) XDR spectra of NANO1, NANO2, and SiQDsMNsSH. 

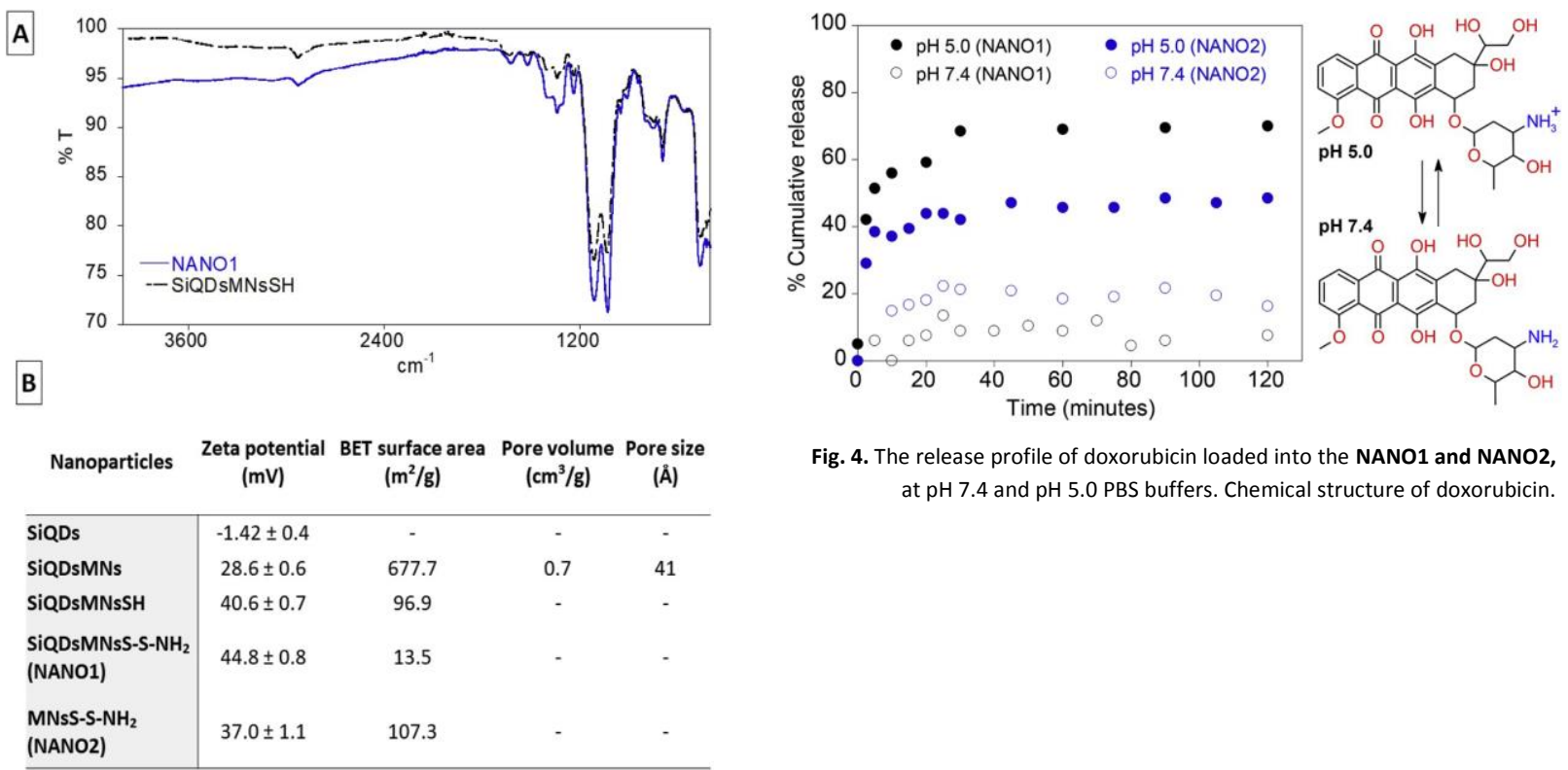

Fig. 4. The release profile of doxorubicin loaded into the NANO1 and NANO2, at $\mathrm{pH} 7.4$ and $\mathrm{pH}$ 5.0 PBS buffers. Chemical structure of doxorubicin.

Fig. 3. (A) FTIR spectra of SiQDsMNsSH and NANO1 nanoparticles. (B) Zeta potential, BET surface, pore volume and size data of the nanoparticles.

\subsection{Doxorubicin loading/release}

Doxorubicin, a common drug used in cancer treatment, was chosen as a model drug to investigate the potential application of the luminescent nanocarrier NANO1. Doxorubicin loading into NANO1 and NANO2 was performed according to section 2.4, and from equations (1) and (2) were obtained encapsulation efficiencies of $41 \%-43 \%$ and loading capacities of $18 \mathrm{mg}$ and $20 \mathrm{mg}$ of doxorubicin per gram for NANO1 and NANO2, respectively. According to the obtained results, the total coverage of NANO1's surface and the presence of a core of SiQDs, led to a reduction in the system's mesoporosity, implying that DOX interactions and loadings occur mostly at the nanoparticles' surfaces via strong electrostatic and hydrogen bonding. In NANO2, despite surface coverage, the absence of a SiQD core, lead to a slightly higher surface area, with some DOX also adsorbing onto the pores' walls, thus justifying the marginally superior loading ratio. Drug release assays were performed in PBS at two different conditions: $\mathrm{pH} 7.4$ and pH 5.0 (Fig. 4). Whilst at pH 7.4 no significant drug release was observed, for NANO1 (ca. 5\%), this phenomenon was favoured at pH 5.0 where release percentages of ca. $70 \%$ were obtained. As previously reported for mesoporous silica nanoparticles [46], a burst release of DOX (5 min) was observed for NANO1. In contrast, NANO2 release percentages of ca. $20 \%$ and $50 \%$, lower than those of NANO1, were obtained at pH 7.4 and pH 5.0 only after 30 min of continuous release. The observed release behaviour is partly attributed to DOX solubility differences, and the attractive - repelling forces developed between the drug and the modified nanoparticles at the respective $\mathrm{pH}$ values. The nanoparticles have a positive charge, while DOX is positively charged at low pH and partly neutral at physiological pH conditions (pKa $1 / 4$ 8.25). The repulsion between DOX and the organic coating and that of DOX with the pores' wall is different. The repulsion at acidic $\mathrm{pH}$ is higher between the positively charged DOX and the positively charged surface coating, which justifies the higher percentage release in NANO1 at pH 5.0. Thus, the release of DOX from the NANO1 nanocarrier is favoured by acidic conditions (Fig. 4).

\subsection{Dose-dependent of cytotoxicity of nanoparticles}

The viability of cell cultures, after exposure to free doxorubicin and the different DOX-loaded, or non-loaded, nanoparticles, was examined using PrestoBlue (Cell viability reagent [47]). The assay measures the amount of resazurin reduction by mitochondrial dehydrogenase and assumes that cell viability (corresponding to reductive activity) is proportional to the production of resorufin which is measured spectrophotometrically $(570 \mathrm{~nm})$, or fluorescently, by exciting at $531 \mathrm{~nm}$ and collecting the emission at $615 \mathrm{~nm}$. Exponentially dividing cells were treated with the different concentrations (from 1 to $300 \mu \mathrm{g} / \mathrm{mL}$ ) of each nanoparticle for $72 \mathrm{~h}$, as described in the experimental section. Control DOX-treated cells were exposed to $1.75 \mu \mathrm{M}$ of doxorubicin to simulate the equivalent concentration of the drug contained inside the tested nanoparticles. The absence of the cytotoxicity of NANO1 and NANO2 can be observed in Fig. 5. No significant effect on cell viability was detected at concentrations up to $75 \mu \mathrm{g} / \mathrm{mL}$ and $150 \mu \mathrm{g} / \mathrm{mL}$, in tumoral cell lines HCT116 and HT29, respectively. Fig. 6 shows the dose-response effect of the two cancer cells lines against free DOX, NANO1@DOX and NANO2@DOX nanoparticles (NPs). As expected for all cell lines, we can confirm that as DOX concentration increased, cell survival decreased. Indicating, thus, that the effect of both free drug and drug-loaded NPs is concentration-dependent. ANO2@DOX and DOX show similar cytotoxic behaviours. With, IC50 values of free DOX and NANO2@DOX NPs being very similar: $1.54 \pm 0.13$ and $0.75 \pm 0.28$ for DOX; and $0.37 \pm 0.02$ and $2.36 \pm 0.02 \mu \mathrm{M}$ for NANO2@DOX. Conversely, NANO1@DOX showed less cytotoxicity, with IC50 values of $8.92 \pm 0.87$ and $9.35 \pm 0.84 \mu \mathrm{M}$, respectively (Table 1). 
HCT116

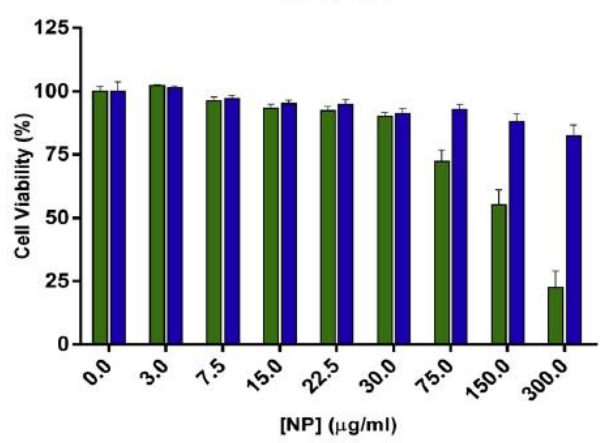

HT29

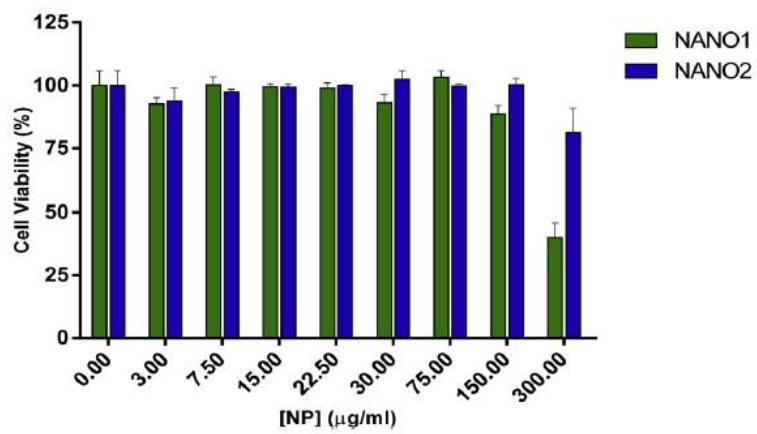

Fig. 5. Effect of NANO1 and NANO2 in cell viability. Viability of HCT116 and HT29 cells was evaluated after 72h exposure to increasing doses of NANO1 and NANO2 NPs. Values are the mean \pm SEM from three independent experiments.
HCT116

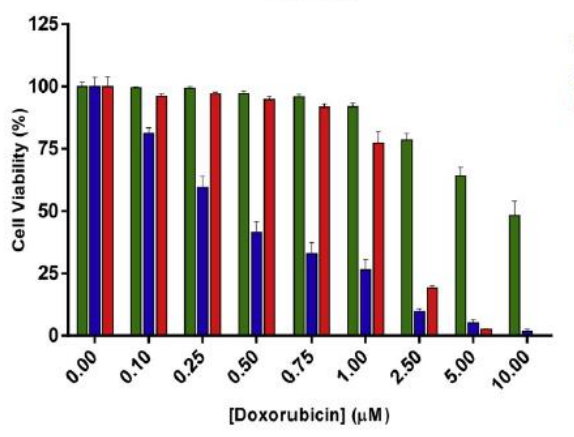

HT29

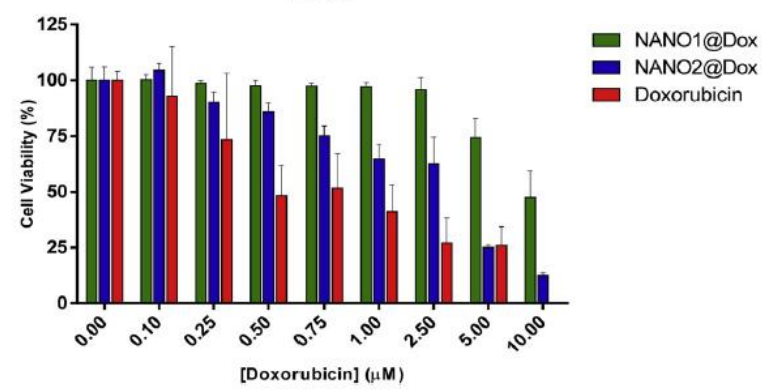

Fig. 6. Effect of NANO1@DOX, NANO2@DOX and doxorubicin in cell viability. Viability of HCT116 and HT29 cells was evaluated after $72 \mathrm{~h}$ exposure to increasing doses of NANO1@DOX.NANO2@DOX NPs and DOX. Values are the mean \pm SEM from three independent experiments.

Table 1

IC $_{50}$ values of DOX and DOX-loaded NPs against HCT116 and HT29 cells. Depicted concentrations (in $\mu \mathrm{M}$ ) correspond to that of free and loaded DOX.

\begin{tabular}{lll}
\hline Nanoparticles/Drugs & \multicolumn{2}{l}{$\mathrm{h}$} \\
\cline { 2 - 3 } & $\mathrm{IC50}(\mu \mathrm{M}) \mathrm{HCT116}$ & $\mathrm{IC50}(\mu \mathrm{M}) \mathrm{HT} 29$ \\
\hline NANO1@DOX & $8.92 \pm 0.87$ & $9.35 \pm 0.84$ \\
NANO2@DOX & $0.37 \pm 0.02$ & $2.36 \pm 0.81$ \\
DOX & $1.54 \pm 0.13$ & $0.75 \pm 0.28$ \\
\hline
\end{tabular}

\subsection{Cellular uptake of doxorubicin-loaded nanoparticles}

After $24 \mathrm{~h}$ of exposure to nanoparticles, cells were stained and imaged by LSCM. Analysis of z-stack images (Fig. 7 B and C) and their orthogonal sectioning demonstrated that both NANO1@DOX and NANO2@DOX are internalized by HT29 cells (Fig. 7 D and E). The red fluorescence from doxorubicin is mostly localized in the cytoplasm, between the blue fluorescence of Hoechst, that signals nuclei position in cells and the green fluorescence of cellular membrane staining. From these images, we can see that DOX was internalized by the cells, arriving at the nuclei of some of them. The fluorescence of SiQDs contained within NANO1 and NANO1@DOX, as well as DOX fluorescence from NANO1@DOX and NANO2@DOX, inside the cells, were quantified by analysing cell lysates in a spectrofluorometer (excitation wavelengths: 370 and $490 \mathrm{~nm}$; emission wavelengths: 499 and $589 \mathrm{~nm}$ respectively for SiQDs and DOX). Table 2 displays the relative cellular internalisation, in percentage, of added nanoparticles and DOX to the cell culture medium. It is interesting to notice that the magnitude of detected fluorescence for DOX, in the case of both nanoparticles, is higher than that of free drug. The relative cellular internalisation of NANO1 and NANO1@DOX was around 30\%, with values for the calculated uptake, at $370 \mathrm{~nm}$ for the SiQDs and $470 \mathrm{~nm}$ for the DOX, being very similar. The percentage of uptake obtained for NANO2@DOX was around 24\%, which is slightly lower than the uptake obtained for NANO1@DOX. In the attempt to correlate the obtained uptakes and cytotoxicity, we can affirm that, although, with the lowest uptake percentages, DOX and NANO2@DOX have the most significant cytotoxic effects against cancer cell lines, with small differences in terms of IC50. While DOX, with the lowest uptake (ca. 14\%), has the best IC50 for HT29 cells, on the other hand, NANO2@DOX with a higher uptake showed outstanding and unmatched cytotoxicity against HCT116 cells. Considering NANO1@DOX high uptake but low cytotoxicity, and according to a fluorescence evaluation-based uptake, it is possible that for NANO1@DOX we are 
detecting fluorescence of DOX that is attached to the nanoparticles. Not being a free drug, DOX-NP complexes lack the capacity to arrive at its target and induce cellular death, within the in vitro tested timescale. Suggesting a possible NANO1@DOX controlled release of DOX, overtime (for $\mathrm{t}>72 \mathrm{~h}$ ).

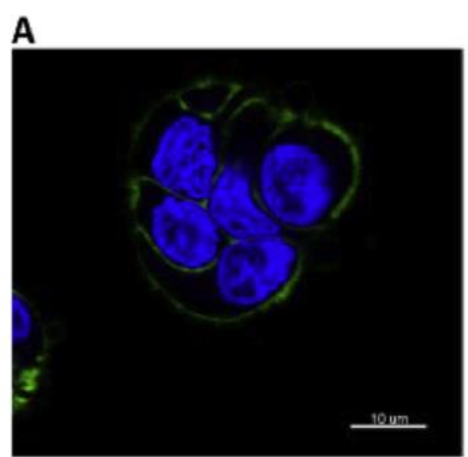

D

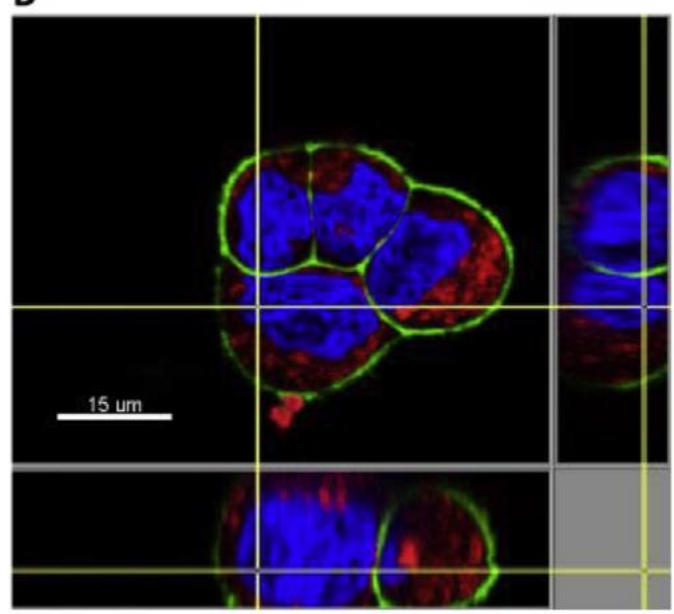

B

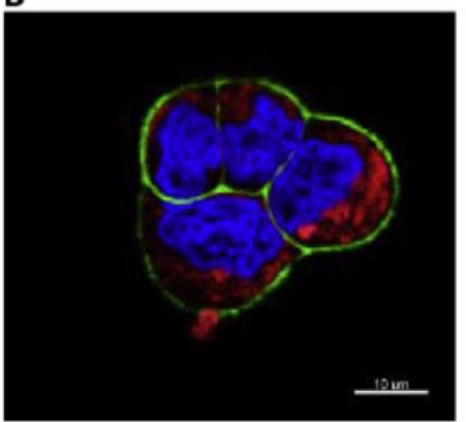

$\mathbf{E}$

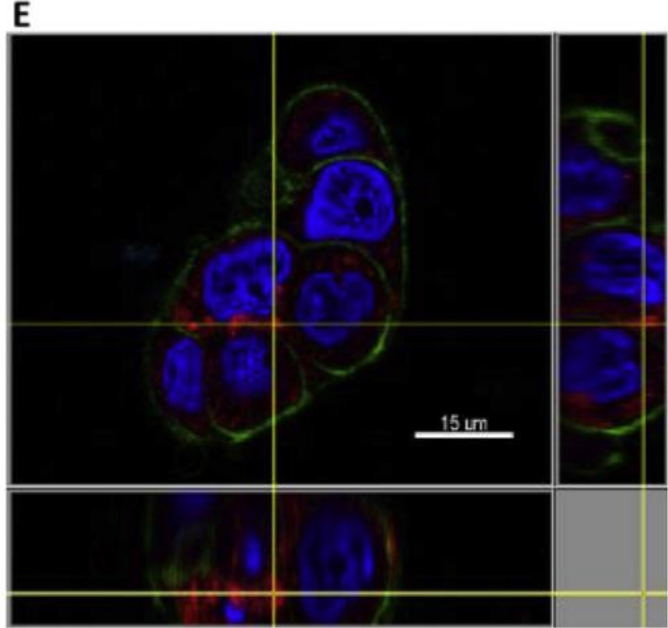

Fig. 7. Laser Scanning Confocal Microscopy (LSCM) in vivo images of control cells (A) and internalized, NANO1@Dox (B and D), and NANO2@Dox (C and E) in HT29 cells. Confocal images obtained from single channels of nanoparticle with doxorubin fluorescence (B-D - red), nuclearStain (Hoeschst- Blue), and membrane stain (CellMask-green) are shown. Orthogonal sectioning and 3D reconstruction of the various z-stacks are shown in D and E images. (For interpretation of the references to color in this figure legend, the reader is referred to the Web version of this article.)

Table 2

Cellular quantification of nanoparticles uptake.

\begin{tabular}{llll}
\hline Nanoformulation/Drug & Uptake & & Uptake \\
\cline { 2 - 2 } & $\left(\lambda_{\text {ex }}=370 \mathrm{~nm}\right)$ & & $\left(\lambda_{\text {ex }}=470 \mathrm{~nm}\right)$ \\
\hline NANO1 & $29,94 \% \pm 1,20 \%$ & & N/A \\
NANO1@DOX & $32,33 \% \pm 12,28 \%$ & & $34,50 \% \pm 2,28 \%$ \\
NANO2 & N/A & & N/A \\
NANO2@DOX & N/A & $24,06 \% \pm 0,39 \%$ \\
DOX & N/A & $14,04 \% \pm 0,79 \%$ \\
\hline
\end{tabular}

\section{Conclusions}

In this study, DOX-loaded nanoparticles based on silica were successfully synthesised, with sizes around $70-80 \mathrm{~nm}$, with $\mathrm{pH}$-dependent behaviours. The in vitro biological effects of the DOX loaded nanoparticles were evaluated on human cancer cell lines of colon. The results indicate that doxorubicin-loaded NPs induced noticeably cytotoxicity and cellular uptake on cancer cells with cytotoxic effects comparable to that of free drugs. The luminescent nanocarrier herein presented (NANO1) showed low toxicity and very promising results on the load and release of DOX, while keeping its fluorescent in cellular environment. Based on these results we envisage the addition of a targeting molecule at the surface of this nanocarrier, in order to make it more selective for a specific type of cell, increasing its range of applications and specificity, as well as promoting a decrease in prescribed DOX, and anti-cancer drugs, in cancer therapies. 
Declaration of competing interest The authors declare that they have no known competing financial interests or personal relationships that could have appeared to influence the work reported in this paper.

CRediT authorship contribution statement Gonçalo A. Marcelo: Validation, Investigation, Data curation, Visualization, Writing - original draft, Writing - review \& editing. David Montpeyo: Validation, Data curation, Investigation. Fernando Novio: Validation, Investigation, Resources, Writing - original draft, Writing - review \& editing, Funding acquisition. Daniel Ruiz-Molina: Resources, Writing - original draft, Writing - review \& editing, Funding acquisition. Elisabete Oliveira: Conceptualization, Methodology, Validation, Investigation, Resources, Data curation, Writing - original draft, Writing - review \& editing, Funding acquisition, Visualization, Supervision, Project administration.

Acknowledgements This work was supported by the Associate Laboratory Research Unit for Green Chemistry - Clean Processes and Technologies - LAQV which is financed by national funds from FCT/MEC (UID/QUI/50006/2013) and co-financed by the ERDF under the PT2020 Partnership Agreement (POCI-01-0145-FEDER- 007265); by the Scientific Society PROTEOMASS (Portugal) (General Funding Grant) and by the Spanish Ministry of Science, Innovation and Universities project RTI2018-098027-B-C22. This work was also supported by grant RTI2018-098027-B-C21 and RTI2018-088027-B-C22 from the Spanish Government funds and by the European Regional Development Fund (ERDF). The ICN2 is funded by the CERCA programme/Generalitat de Catalunya. The ICN2 is supported by the Severo Ochoa Centres of Excellence programme, funded by the Spanish Research Agency (AEI, grant no. SEV-2017-0706). F. N thanks to COST Action CA17121. F. N and J. L thanks to COST Action CA15138. G.M thanks to FCT/MEC (Portugal) for his doctoral grant PD/ BD/142865/2018. E. O thanks FCT/MEC (Portugal) for the individual contract, CEECIND/00648/2017.

\section{Appendix A. Supplementary data}

Supplementary data to this article can be found online

\section{References}

[1] Ferlay Js I, Ervik M, Dikshit R, Eser S, Mathers C, Rebelo M, Parkin DM, Forman D, Brav F. Cancer incidence and mortality worldwide: JARC CancerBase No11. GLOBOCAN; 2012. [2] Chauhan VP, Jain RK. Strategies for advancing cancer nanomedicine. Nat Mater 2013;12(11):958-62.

[3] Maeda H, Nakamura H, Fang J. The EPR effect for macromolecular drug delivery to solid tumors: improvement of tumor uptake, lowering of systemic toxicity, and distinct tumor imaging in vivo. Adv Drug Deliv Rev 2013;65(1):71-9.

[4] Liu D, Yang F, Xiong F, Gu N. The smart drug delivery system and its clinical potential. Theranostics 2016;6(9):1306-23.

[5] Chan MH, Lin HM. Preparation and identification of multifunctional mesoporous silica nanoparticles for in vitro and in vivo dual-mode imaging, theranostics, and targeted tracking. Biomaterials 2015;46:149-58.

[6] Kong M, Tang J, Qiao Q, Wu T, Qi Y, Tan S, et al. Biodegradable hollow mesoporous silica nanoparticles for regulating tumor microenvironment and enhancing antitumor efficiency. Theranostics 2017;7(13):3276-92.

[7] Wang Q, Bao Y, Ahire J, Chao Y. Co-encapsulation of biodegradable nanoparticles with silicon quantum dots and quercetin for monitored delivery. Adv Healthc Mater 2013;2(3):45966 .

[8] Chan WC, Nie S. Quantum dot bioconjugates for ultrasensitive nonisotopic detection. Science 1998;281(5385):2016-8.

[9] Erogbogbo F, Yong KT, Roy I, Xu G, Prasad PN, Swihart MT. Biocompatible luminescent silicon quantum dots for imaging of cancer cells. ACS Nano 2008;2(5): 873-8.

[10] Lu Y, Zhong Y, Wang J, Su Y, Peng F, Zhou Y, et al. Aqueous synthesized near-infrared-emitting quantum dots for RGD-based in vivo active tumour targeting. Nanotechnology 2013;24(13). 135101.

[11] Park JH, Gu L, von Maltzahn G, Ruoslahti E, Bhatia SN, Sailor MJ. Biodegradable luminescent porous silicon nanoparticles for in vivo applications. Nat Mater 2009;8 (4):331-6.

[12] Delley B, Steigmeier EF. Quantum confinement in Si nanocrystals. Phys Rev B Condens Matter 1993;47(3):1397-400.

[13] Wilson WL, Szajowski PF, Brus LE. Quantum confinement in size-selected, surface-oxidized silicon nanocrystals. Science 1993;262(5137):1242-4.

[14] Cheng X, Lowe SB, Reece PJ, Gooding JJ. Colloidal silicon quantum dots: from preparation to the modification of self-assembled monolayers (SAMs) for bio-applications. Chem Soc $\operatorname{Rev} 2014 ; 43(8): 2680-700$.

[15] Zhong Y, Peng F, Bao F, Wang S, Ji X, Yang L, et al. Large-scale aqueous synthesis of fluorescent and biocompatible silicon nanoparticles and their use as highly photostable biological probes. J Am Chem Soc 2013;135(22):8350-6.

[16] Zhong Y, Sun X, Wang S, Peng F, Bao F, Su Y, et al. Facile, large-quantity synthesis of stable, tunable-color silicon nanoparticles and their application for long-term cellular imaging. ACS Nano $2015 ; 9(6): 5958-67$

[17] Li Q, He Y, Chang J, Wang L, Chen H, Tan YW, et al. Surface-modified silicon nanoparticles with ultrabright photoluminescence and single-exponential decay for nanoscale fluorescence lifetime imaging of temperature. J Am Chem Soc 2013;135 (40):14924-7.

[18] Wang J, Ye D-X, Liang G-H, Chang J, Kong J-L, Chen J-Y. One-step synthesis of water-dispersible silicon nanoparticles and their use in fluorescence lifetime imaging of living cells. J Mater Chem B 2014;2(27):4338-45.

[19] Ma SD, Chen YL, Feng J, Liu JJ, Zuo XW, Chen XG. One-step synthesis of water-dispersible and biocompatible silicon nanoparticles for selective heparin sensing and cell imaging. Anal Chem 2016;88(21):10474-81.

[20] Mura S, Nicolas J, Couvreur P. Stimuli-responsive nanocarriers for drug delivery. Nat Mater 2013;12(11):991-1003.

[21] Marcelo G, Ariana-Machado J, Enea M, Carmo H, Rodriguez-Gonzalez B, Luis Capelo J, et al. Toxicological evaluation of luminescent silica nanoparticles as new drug nanocarriers in different cancer cell lines. Materials 2018;11(8):1310.

[22] da Paz MC, Santos Mde F, Santos CM, da Silva SW, de Souza LB, Lima EC, et al. Anti-CEA loaded maghemite nanoparticles as a theragnostic device for colorectal cancer. Int J Nanomed 2012;7:5271-82.

[23] Vigor KL, Kyrtatos PG, Minogue S, Al-Jamal KT, Kogelberg H, Tolner B, et al. Nanoparticles functionalized with recombinant single chain Fv antibody fragments (scFv) for the magnetic resonance imaging of cancer cells. Biomaterials 2010;31 (6):1307-15.

[24] Tiernan JP, Ingram N, Marston G, Perry SL, Rushworth JV, Coletta PL, et al. CEA-targeted nanoparticles allow specific in vivo fluorescent imaging of colorectal cancer models. Nanomedicine 2015;10(8):1223-31.

[25] Fay F, McLaughlin KM, Small DM, Fennell DA, Johnston PG, Longley DB, et al. Conatumumab (AMG 655) coated nanoparticles for targeted pro-apoptotic drug delivery. Biomaterials 2011;32(33):8645-53.

2011;32(33):8645-53. [26] Abdelghany SM, Schmid D, Deacon J, Jaworski J, Fay F, McLaughlin KM, et al. Enhanced antitumor activity of the photose
tosylate through encapsulation in antibody-targeted chitosan/alginate nanoparticles. Biomacromolecules 2013;14(2):302-10.

[27] Cortez C, Tomaskovic-Crook E, Johnston AP, Scott AM, Nice EC, Heath JK, et al. Influence of size, surface, cell line, and kinetic properties on the specific binding of A33 antigentargeted multilayered particles and capsules to colorectal cancer cells. ACS Nano 2007;1(2):93-102.

[28] Kirui DK, Rey DA, Batt CA. Gold hybrid nanoparticles for targeted phototherapy and cancer imaging. Nanotechnology 2010;21(10). 105105.

[29] Yang SJ, Lin FH, Tsai KC, Wei MF, Tsai HM, Wong JM, et al. Folic acid-conjugated chitosan nanoparticles enhanced protoporphyrin IX accumulation in colorectal cancer cells. Bioconjugate Chem 2010;21(4):679-89.

[30] Li P, Wang Y, Zeng F, Chen L, Peng Z, Kong LX. Synthesis and characterization of folate conjugated chitosan and cellular uptake of its nanoparticles in HT-29 cells. Carbohydr Res 2011;346(6):801-6.

[31] Kopansky E, Shamay Y, David A. Peptide-directed HPMA copolymer-doxorubicin conjugates as targeted therapeutics for colorectal cancer. J Drug Target 2011;19 (10):933-43.

[32] Unzueta U, Cespedes MV, Ferrer-Miralles N, Casanova I, Cedano J, Corchero JL, et al. Intracellular CXCR4(b) cell targeting with T22-empowered protein-only nanoparticles. Int J Nanomed 2012;7:4533-44.

[33] Jain A, Jain SK, Ganesh N, Barve J, Beg AM. Design and development of ligand-appended polysaccharidic nanoparticles for the delivery of oxaliplatin in colorectal cancer. Nanomedicine 2010;6(1):179-90.

[34] Gary-Bobo M, Brevet D, Benkirane-Jessel N, Raehm L, Maillard P, Garcia M, et al. Hyaluronic acid-functionalized mesoporous silica nanoparticles for efficient photodynamic therapy of cancer cells. Photodiagnosis Photodyn Ther 2012;9(3): 256-60. 
[35] Shahzad MM, Mangala LS, Han HD, Lu C, Bottsford-Miller J, Nishimura M, et al. Targeted delivery of small interfering RNA using reconstituted high-density lipoprotein nanoparticles. Neoplasia 2011;13(4):309-19.

[36] Stan MS, Sima C, Dinischiotu A. Silicon quantum dots: from synthesis to bioapplications. Bioactivity of Engineered Nanoparticles2017. p. 339-359.

[37] Marcelo GA, Lodeiro C, Capelo JL, Lorenzo J, Oliveira E. Magnetic, fluorescent and hybrid nanoparticles: from synthesis to application in biosystems. Mater Sci Eng C Mater Biol Appl 2020;106:110104

[38] Xu K, Wang J-X, Kang X-L, Chen J-F. Fabrication of antibacterial monodispersed Ag-SiO2 core-shell nanoparticles with high concentration. Mater Lett 2009;63(1): 31-3.

[39] Lai CY, Trewyn BG, Jeftinija DM, Jeftinija K, Xu S, Jeftinija S, et al. A mesoporous silica nanosphere-based carrier system with chemically removable CdS nanoparticle caps for stimuliresponsive controlled release of neurotransmitters and drug molecules. J Am Chem Soc 2003;125(15):4451-9.

[40] Brunauer S, Emmett PH, Teller E. Adsorption of gases in multimolecular layers. J Am Chem Soc 1938;60(2):309-19.

[41] Kellogg RE, Bennett RG. Radiationless intermolecular energy transfer. III. Determination of phosphorescence efficiencies. J Chem Phys 1964;41(10):3042-5.

[42] Tu J, Boyle AL, Friedrich H, Bomans PH, Bussmann J, Sommerdijk NA, et al. Mesoporous silica nanoparticles with large pores for the encapsulation and release of proteins. ACS Appl Mater Interfaces 2016;8(47):32211-9.

[43] Dasog M, Yang Z, Regli S, Atkins TM, Faramus A, Singh MP, et al. Chemical insight into the origin of red and blue photoluminescence arising from freestanding silicon nanocrystals. ACS Nano 2013;7(3):2676-85

[44] Dasog M, De los Reyes GB, Titova LV, Hegmann FA, Veinot JG. Size vs surface: tuning the photoluminescence of freestanding silicon nanocrystals across the visible spectrum via surface groups. ACS Nano 2014;8(9):9636-48.

[45] Hou X, Xu H, Pan L, Tian Y, Zhang X, Ma L, et al. Adsorption of bovine serum albumin on superparamagnetic composite microspheres with a Fe3O4/SiO2 core and mesoporous SiO2 shell. RSC Adv 2015;5(126):103760-6.

[46] Oliveira E, Santos HM, Jorge S, Rodríguez-Gonz alez B, Novio F, Lorenzo J, et al. Sustainable synthesis of luminescent CdTe quantum dots coated with modified silica mesoporous nanoparticles: towards new protein scavengers and smart drug delivery carriers. Dyes Pigments 2019;161:360-9.

[47] Xu M, McCanna DJ, Sivak JG. Use of the viability reagent PrestoBlue in comparison with alamarBlue and MTT to assess the viability of human corneal epithelial cells. J Pharmacol Toxicol Methods 2015;71:1-7. 